Herbert Bassarak (Hrsg. 2018):

\title{
Lexikon der Schulsozialarbeit
}

1. Auflage, Nomos-Verlag, Baden-Baden, 634 Seiten, $€ 98$,-

\section{Aktuell und praxisnah:}

Umfassendes Nachschlagewerk

für Soziale Arbeit an Schulen

Ein Lexikon der besonderen Art hat Prof. Herbert Bassarak herausgegeben. Denn er konnte gut 200 Autorinnen und Autoren - davon der überwiegende Teil praktizierende Sozialarbeiterinnen und Sozialarbeiter an Schulen aus dem deutschsprachigen Raum - gewinnen, welche mehr als 450 relevante Stichworte aus dem weiten Feld der Schulsozialarbeit bearbeitet haben. Die einzelnen Beiträge umfassen jeweils ein bis zwei Seiten und bestechen durch ihre ungemeine Praxisrelevanz und Fachlichkeit sowie ihre ausgesprochene Lesefreundlichkeit. Dabei werden vielseitige Fragestellungen zu den Bildungs- und Erziehungssystemen erörtert und zugleich wird damit der so nötige wissenschaftstheoretische Diskurs geführt. In diesem Pendel zwischen der Praxis im Handlungsfeld und den Rahmenbedingungen struktureller Art bewegen sich die von den Autorinnen und Autoren selbstverantwortlich verfassten Beiträge, welche naturgemäß zum Teil auch kontroverse Standpunkte beinhalten. Doch gerade diese Verknüpfung der praktischen und theoretischen Gesichtspunkte aus unterschiedlichen Disziplinen und praktischen Erfahrungen machen den besonderen Charme dieses Lexikons aus. Im ersten Beitrag wird deutlich, dass die „Abgrenzung Jugendsozialarbeit an Schulen und Schulsozialarbeit" nicht immer ganz leicht fällt und Schulsozialarbeit eher die gesamte Schülerschaft betrifft, während sich Jugendsozialarbeit an Schulen an spezifisch beeinträchtigte, sozial benachteiligte Kinder und Jugendliche richtet. Doch in der Praxis spielen andere Punkte eine wesentliche Rolle. Dies wird spätestens bei den Beiträgen zu dem Thema Zwangsheirat, Pubertät, Selbstverletzendes Verhalten, Trennungs- und Scheidungsberatung, Kindeswohlgefährdung oder Schweigepflicht deutlich, wenn ausgesprochen praxisbezogen geschrie- ben wird, ohne sich in kleinteiligen Punkten zu verlieren. Rechtliche, psychologische, sozialarbeiterische, methodische und kulturelle Aspekte fließen in den Texten ausführlich ein. Diverse besondere Präventionsangebote werden genauso vorgestellt wie auch fachpraktische Hinweise gegeben und einzelne Erfahrungen mit Projekten und Medien dargestellt. Mal liegt der Fokus mehr direkt auf den schulischen Kontext betreffende Punkte, mal sind es stärker Aspekte, die eher der Jugendhilfe zuzuordnen sind. Die konzeptionelle Vielfalt der Sozialarbeit an Schulen wird bei der Lektüre ausgesprochen deutlich. Ob nun Sucht, Gewalt, Elternarbeit, Sozialraum, Schutzkonzepte, Sozialtrainings, Suizidprävention, Cybermobbing, Mädchenarbeit, Essstörungen oder Ganztagsschule, politische Bildung oder Berufsorientierung: Hier kann jede Fachperson der Sozialen Arbeit an Schulen aus dem reichen Fundus an Erfahrungen aus der praktischen Arbeit bzw. der entsprechenden Reflexion schöpfen. Das zweispaltige Textlayout macht das Lexikon sehr übersichtlich und wird durch den Fettdruck der Stichwörter unterstützt.

Die Anzahl an Literaturhinweisen ist bei den einzelnen Beiträgen begrenzt. Dafür gibt es eine große Anzahl von binnenstrukturellen Querverweisen und ein ausgezeichnetes 75-seitiges, oft sehr aktuelle Titel berücksichtigendes Literaturverzeichnis. Beim Stichwortverzeichnis wird leider nicht angegeben, wer die jeweilige Autorenschaft verantwortet. Dafür gibt es aber ein Verzeichnis der Autorinnen und Autoren, bei welchem die beruflichen Hintergründe und Emailanschriften aufgeführt sind. So ließe sich gegebenenfalls auch Kontakt zu den einzelnen Personen aufnehmen. Die Komplexität des Arbeitsfeldes und der hohe Anspruch, der an die Schulsozialarbeiterinnen und Schulsozialarbeiter gestellt 
wird, macht dieses Lexikon noch einmal überdeutlich. Im Gesamten ist dieses Lexikon eine ausgesprochen vielseitige, gut strukturierte Ansammlung an Informationen und fachlichen Einschätzungen. Es kann damit gut zur weiteren Professionalisierung des so zukunftsträchtigen Praxisfeldes beitragen und helfen, dass Schul- sozialarbeit durch fundierte Konzepte und eine verlässliche Ausstattung an Personal und Räumen in ihrer Wirksamkeit gestärkt wird.

Detlef Rüsch

E-Mail:detlefruesch@aol.com

DOI 10.2378/uj2019.art15d 